\title{
12
}

\section{Emotional Textual Analysis}

\author{
RENZO CARLI, ROSA MARIA PANICCIA, FIAMMETTA GIOVAGNOLI, \\ AGOSTINO CARBONE, AND FIORELLA BUCCI
}

$\mathrm{I}_{\mathrm{n}}^{\mathrm{n}}$ n this chapter, we present a psychological methodology called emotional textual analysis (ETA; Carli \& Paniccia, 2002). Much of this work is embedded within more psychoanalytic methods, which might be somewhat foreign to those involved in community-based research. In brief, when using ETA, texts are collected and analyzed with the aim of using the meaning of the words to provide knowledge to ultimately design culturally appropriate interventions. The method is based on the study of the association of dense words, that is, words that when taken out of the context of discourse have an immediate, very strong emotional sense- (e.g., bomb, mother, travel). In this approach, language is thought of as an organizer of the relationship between the individual contributor of the text and his or her context, rather than just an expression of the individual's emotions. Tracks of these written representations are viewed within the complexity of relationships and settings.

ETA can be used to explore the culture of organizations in the production and service sectors, as well as the cultures characterizing many social and community groups. We refer to the culture identified using this methodology as local culture. ETA analyses are calibrated to the local cultures and have been used as a basis for bringing about interventions. Many examples of this method's use in Italy are described elsewhere (e.g., Paniccia, Giovagnoli, Bucci, \& Caputo, 2014; Paniccia, Giovagnoli, \& Caputo, 2014) and, more generally, in the e-journal Rivista di Psicologia Clinica.

In this chapter, we first provide the theoretical models upon which ETA is based. We discuss how, in its reliance on the assessment of both emotions and relationships, it differs from other qualitative approaches. We then provide a case example in which the application of ETA facilitated the explication of the employment situation of workers in Italy.

\section{INTRODUCTION TO EMOTIONAL TEXTUAL ANALYSIS \\ Models Underlying Emotional Textual Analysis}

ETA draws from specific traditions of psychological theories. Gestalt theory is one primary frame of reference. It stresses that we actively confer meaning to stimuli through a lens that depends on our culture, the context in which we grew up, and the context in which we perceive the world. Central to ETA is Freud's conceptualization of the unconscious. Matte Blanco (1975) suggested that the unconscious is a mode of thinking of the mind characterized by a symmetrical logic, unlike the logic of conscious thinking. According to Matte Blanco (1975), we operate continually with paradoxes, with schizophrenic thought being an extreme example. Schizophrenic thought seems strange to us, but it might be logical for symmetrical logic because external reality has been replaced by an inner, psychological reality. In this view, thought is always a compromise between these two forms of logic, unconscious and conscious thought. In the ETA approach, the unconscious is seen as the attribution of emotional meaning to social and contextual reality.

ETA also draws from another psychoanalytic tradition, object relations theory, which asserts that that we attribute emotional significance to every dimension of reality with which we relate and that we interpret these emotional experiences as being intentioned toward us. For example, a child may 
bang his or her head against the table and say "Bad table!" The table is viewed as having intentionally inflicted pain on the child. This process is present in adults as well as children. Adults sometimes get upset with a computer that does not obey their commands, despite knowing that the computer is inanimate and not capable of purposefully ignoring commands. We grow up giving emotional meaning to all aspects of reality that we encounter, such as the mother's nipple, light, sound, a voice, or a word.

The elaboration of ETA started in the 1970s within a multivocal cultural context. The publication of "The Unconscious as Infinite Sets" by Matte Blanco (1975) and the book Symbol and Code by Fornari (1976) were important contributions in the development of our theory of emotional symbolization. In this vein, in connection with French psycho-sociology and the journal Connexion (http://www.cairn.info/revue-connexions), we began to study social relationships and the dynamics of power in real social groups in the field, such as youth groups, national and international companies (e.g., electricity and communications companies, the Italian Banking Association), small and medium-sized enterprises, schools, and health services. The idea that thinking is always a compromise between the two forms of logic, that of the unconscious and that of the conscious, was applied to a psychological theory of social relationships.

In the ETA approach, the purpose of psychological interventions is to enable the client to conceive of and interpret the collusive dynamics that are operating in a particular context. By collusive dynamics, we mean the emotional symbolization of the context socially shared by those who belong to it. The social relationship originates from the shared emotional symbolization of the context, which forms the basis of sense making and behavior regulation in social groups. We attribute emotional significance to every dimension of reality with which we relate. However, because it is based on the symmetrical logic of the unconscious, emotional symbolization tends to assimilate and confuse external reality with internal, psychic reality. Only the thinking encompassed by emotional symbolization allows one to recover the distinction between external reality, on the one hand, and the multiple, different meanings that we can attach to it in our subjective representation, on the other. The process of thinking of the collusive dynamics - that is, the shared emotional symbolization of the context within, for example, a group, an organization, or a community - is the key goal of ETA and, more generally, of the psychological intervention. By exploring the most significant criticalities and resources which are present in the emotional dynamics that underlie social relationships_-such as dynamics of power, dependency, and achievement (Carli \& Paniccia, 2002)_ETA improves the client's organizational competence and supports social development.

In order to organize the research and interpret the collusive dynamics expressed by a text (e.g., a set of interview transcripts), it is essential to consider the relationships within which the text was produced. We need to consider in which social groups we collected the text and what goals we have for the analyses. In this method it is important to identify the people who requested assistance and to keep them in mind throughout the research process. In other words, when collecting and interpreting this type of data, a researcher needs to be clear about who will use them. The relationship is fundamental and influences the way in which research participants are approached, helps identify the models used to explore the data, and provides aid in interpreting findings.

\section{The Double Impact}

\section{of Language: Emotional and Cognitive}

Within this context, words are an essential component of the ETA approach. If we use the subject and the object without the verb, we speak in ways that are hard to understand, such as by saying "I pasta." If the person had said, "I eat pasta," we would understand the meaning. It is evident that if our expressions are not complete, we cannot be understood. In addition, our language, like everything that we experience, has a double meaning, both emotional and cognitive. If a person says, "I devoured that book," we do not think that the person ate a book but that it was read avidly. We understand the meaning of "devoured" by understanding the emotional sense. Additionally, some words have a very strong emotional significance when expressed in specific settings. For example, at a sporting event, a famous soccer player who had an episode of tuberculosis and had just returned to play, heard a fan of the opposing team call him "Lung." The shouted word, in that context, had a clear-cut sense of aggression. It is clear that many words have strong emotional connotations. We can think of a continuum: On the 
extreme left side there are emotionally ambiguous words, which become effective only within their context; they are said to have low polysemy. On the opposite side, words have high polysemy, that is, a minimum of ambiguity because they are emotionally meaningful even when isolated from their discursive context. For example, the verb "to go" has a low emotional profile- "Where are you going tonight?" - and does not indicate emotions except, at times, within a sentence. Completely different is "to go away." Words such as "to go away," "hatred," "failure," and "ambition" are characterized by a maximum of emotional density and a minimum of ambiguity of meaning. We call these words dense words because they are emotionally dense. The emotionally dense sense is evident even when the word is taken out of the context of discourse.

\section{Emotional Textual Analysis as a} Methodology for Textual Analysis

In consulting work for organizations since the 1960s, Carli and Paniccia $(1981,2003)$ have developed a psychological theory and methodology of intervention called the analysis of the demand. This work is based on the analysis of cultures as mediators between individuals and context. The analysis of the demand proposes that interventions should not be focused on correcting deficits but rather on developing the client's resources. Using this approach, psychologists explore the emotional symbolization of problems in order to see how these relate to the client's goals and the possibility of achieving them. It is important to consider that by client we can mean an individual, a social organization, or a community requesting the intervention.

In the mid-1980s, consistent with the theoretical and methodological assumptions of analysis of the demand, Carli and Paniccia (2002) developed the methodology called ETA. This method uses the collection and analysis of texts in order to explore specific topics. Texts are analyzed based on writings, as well as on individual or group interviews. ETA aims to uncover how language expresses emotions-in other words, how language indicates emotional symbolization or the collusive dynamics of contexts. Because the aim is to approach the symmetrical unconscious logic of the text, ETA identifies the dense words within the text. To do this, ETA uses specific software, such as Alceste (Reinert, 1993) or T-LAB (Lancia, 2004), that divides the text collected by the researcher into units of meaning according to special algorithms (Benzècri, 1981).

Using this approach, we could, for example, ask the citizens of a city to provide a response to the question "What do you think of the traffic in your city?" There could be 50 people interviewed, each one having different structural characteristics (e.g., gender, age, socioeconomic level). Using the data collected, the software will generate a dictionary of all the words contained in the text. The researcher then chooses which words to be considered dense. Once the text has been divided into segments and only the dense words have been isolated, it becomes evident that there are segments within which some dense words and not others recur. By using multiple correspondence analysis and cluster analysis, text segments can be placed on the $\mathrm{X}$-axis and the dense words on the Y-axis, allowing some clusters of dense words to be obtained and positioned in a factorial space (for more details, see Bucci, 2014). The clusters' interpretation is governed by the assumption that the co-occurrence of dense words within the text segments highlights the collusive dynamic expressed by the text. The collusive dynamic thus identified is historically situated and characterizes individuals defined within a specific context.

We came to elaborate ETA after years of intervention with organizations of various kinds and conducted research in order to verify if the clusters of dense words actually express the collusive process of the group interviewed. To verify correspondences and mismatches, the textual analysis should be conducted in parallel by more than one person. We have sufficiently communicable criteria, such as the notions of emotional polysemy and ambiguity. It is important to be aware that each person will take interpretative initiatives and declare one's own choices. The reading of the clusters and the dense words may invite intuitive interpretations. In this case, the researcher will find only what she or he already knows. To minimize this, specific models of the relationship formulated by the analysis of the demand which theoretically informs ETA are necessary in order to carry out the clusters' interpretation (Carli \& Paniccia, 2002).

So far we have been speaking mainly about the clusters of dense words. It is important to consider, however, that the reading of the data cannot happen if there is no reference to two other parameters, namely, the factorial space and the relationship with the client who will utilize the 
research findings. When using ETA, one studies the clusters, then relates them to the factorial space, then goes back to the clusters, and so on, in a spiral process. The interpretation takes time. It is also important to keep in mind that the multivariate statistics adopted for ETA do not prove hypotheses but help to build them. This type of research produces interpretative hypotheses of the theme under investigation. It is important to share hypotheses, as well as the verification of these hypotheses, with the citizen or community group requesting the intervention. The following case study illustrates the application of ETA.

\section{CASE STUDY}

Since the end of the 1990s, the Italian labor market has been radically transformed. New forms of atypical employment spread, together with a multiplicity of nonstandard contracts (Fanelli et al., 2006). Atypical employment was meant to develop a flexibility that seemed to be advantageous, as it could accommodate the rapid change of companies' needs and enable people with obligations outside of the workplace (e.g., students, mothers of young children) to access the labor market. The aim was to overcome the excessive rigidity of the traditional employment contracts that hindered companies' hiring plans.

There has been tension within the Italian culture regarding this new form of employment. These new types of employment contracts often have lacked appropriate legal and trade-union protections traditionally important in Italian work culture. Consequently, the labor market has been divided into two parts, with protected workers on the one hand and marginal and insufficiently protected workers on the other. As a consequence, the atypical contracts have created workers who have precarious working conditions. Flexibility has been rapidly transformed into precariousness (Fanelli et al., 2006).

In the research presented here, we interviewed 97 employees having an atypical employment contract. It is important to note that, in ETA, those doing the interviews play an important role in presenting the research and its goals to the respondents. The interviewers are trained in how to select the research participants, share the interviews' aims with interviewees, and support the interviewees' associative process during the interview without interrupting them. In the study with atypical workers, the interviews were based on the following open-ended question: "As members of a university that is engaged in research and interventions concerning problems related to social coexistence, we are interested in flexible work. As you are engaged in flexible work, please tell us everything that comes to your mind when thinking about your work."

The open-ended question is actually not a simple question. During the training, the interviewers often want to know why they are to ask just one question. They frequently express concern that the interviewee's reply might last no more than $5 \mathrm{~min}$ utes. However, as part of this one-question process, interviewers also provide interviewees with information on who the interviewer is and why he or she is asking this question, and are trained to establish a relationship, one that offers the interviewee an opportunity to think about one's own experience by sharing thoughts with another. This method provides an invitation to say anything that crosses the interviewee's mind on the subject. Everything said will be considered relevant. The objective is to follow the thinking of the interviewee without guiding it beyond the initial approach. This procedure is consistent with the exploration of the emotional symbolization of the theme by the interviewee. An interviewer with little experience in this method might think that the interviewee would have no interest in replying for half an hour to "only one question." Conversely, one might also think that a theme could be so emotionally involving for the interviewee that it might be hard to deal with all the generated affect and issues. For example, if the atypical worker interviewed said, "I have had enough of this work, I have been doing this for a long time, I am very angry" and then fell silent, an inexperienced interviewer might be uncertain about what to do next and might ask: "How long have you been doing this job?" In this case, the interviewee might then answer " 15 years" and fall silent again. This interviewer might find herself or himself asking one question after the other, steering the conversation to follow her or his own thoughts on the atypical employment. This would not be a good outcome for the interview. In contrast, a well-trained interviewer would wait before saying anything, without any impatience, looking in a friendly way at the interviewee. If the silence continued, the interviewer would repeat the interviewee's last word in a 
questioning tone: “Angry..." in order to reopen the exchange, without influencing the emotional tone of the interviewee's responses.

Data from the interviews explicated our understanding of the tensions experienced by atypical employees. Specifically, the data revealed which words held especial significance. In this particular study, a central word used by workers was guarantee. The etymological Germanic root of this word means to defend and to protect. A second key word that often appeared in the interviews was future; the use of this word inherently suggests unpredictability. At this point an emotional sense began to unfold. The third key word was the verb project, which means to think of the future. The lack of guarantee is connected to unpredictability when one looks ahead. In this example, we can grasp the fundamental principle of the analysis of the co-occurrences of dense words in the cluster. The co-occurrence of guarantee-future-project is what is meant by word clustering. Data analysis led to the positioning of this cluster on the right polarity of the first factorial axis. In contrast, another cluster ended up, by the data analysis, being positioned on the opposite polarity of the same factor. The first dense words characterizing the second cluster were money and euro. Thus, these two clusters expressed two different and contrasting symbolizations of work that were present in the respondents' discourse. The first emphasized the experience of unpredictability and lack of future associated with work, and the other instead presented work exclusively as a way to make enough money in order to support one's own family.

One soon realizes that within a specific text, some words have a higher relevance in relation to the particular context to which the text refers. For example, the word queue becomes dense if you analyze the culture of citizens with respect to banking services; queuing up at the counter can mark a specific cultural model according to which, for example, the bank is emotionally perceived as an enemy. This kind of research systematically requires the researcher to make specific interpretive choices. The emotional density of a word does not linearly coincide with the frequency of the word within the text. In some cases, for example, a high-frequency word can be so stereotypical for the group interviewed that it does not allow the exploration of any significant differences concerning the emotional symbolization of the investigated problem within the group interviewed.
Another application of ETA is research that we carried out for "Sapienza” University of Rome. The university is located in a working-class neighborhood where there has recently been a rise in antistudent feeling, with police patrolling the streets, students putting up posters, and so on. The aim of the research was to improve the relationship between the university and the neighborhood (Carli \& Pagano, 2008). The knowledge of this local culture allowed us to find cultural resources to promote integration between the two. The ETA process, in such instances as this, enables us to longitudinally explore and potentially verify local cultural changes. Specifically, once a first analysis within a certain context has been made and discussed with those who commissioned it, it is possible to carry out a second analysis after a particular period of time.

This kind of research often generates follow-up initiatives by the organizations requesting the analysis. In such cases, the researcher can give advice on possible actions to be taken, with a new, subsequent ETA carried out to assess follow-up results, if desired. For example, in an ETA examination of the local culture of a mental health center, one cluster indicated the presence of service users presenting problems that could not be classified in psychiatric terms and required a more complex treatment with less predictable outcomes (Paniccia, Di Ninni, \& Cavalieri, 2006). These findings helped initiate a discussion concerning what to do about this new group of patients. Before the ETA analysis there had been little awareness of the differences between clients, but, as a result of using this approach, the mental health center developed an appreciation of the problem.

Similarly, in an ETA assessment in another Italian mental health center, Paniccia, Dolcetti, Giovagnoli, and Sesto (2014) explored the culture of the reception service and investigated requests addressed to mental health services. Discrepancies were found between clients' needs and the services provided, as the mental health center often failed to recognize differences among service users' requests. To address the problems revealed by the study, the center is seeking support in the categorization and differentiation of service users' requests. When using ETA, investigators do not necessarily aim to solve a specific problem but rather to transform the situation or culture from which the problem arose. Illustratively, in this mental health center 
study, one of the foci of discussion of the results with the staff was a dense word cluster which indicated that service providers felt a sort of ideological obligation to give "the right answer" to everyone who came to the mental health center. After some months, the center asked us to extend the investigation through further interviews with service users. Frequently, ETA is able to shed light on minority cultures or points of view. Such analysis is possible if the organization acknowledges that it has a problem and is interested in understanding it instead of perpetuating the habitual collusive dynamics. The intervention is grounded in the possibility that in the relationship between the researcher and the client or citizen group, there is interest in the shared problem.

\section{CONCLUSION}

In this chapter, we have presented ETA as a distinctive way of collecting and interpreting textual data. By analyzing the co-occurrence of dense words within the text, ETA enables us to examine the emotional dynamics underlying relationships within social groups and local communities. As was illustrated by the case examples, the data collected through interviews and analyzed can be used to effect an intervention at a variety of levels.

Of course, one might ask why an investigator does not just keep all the words of the interviewee, rather than selecting from them, as is done in the ETA approach. Our approach aims to examine the symmetrical logic of the unconscious. With ETA we are placed in a space of mediation between the unconscious and the conscious. ETA methodology enables us, by investigating dense words and word clusters, to grasp the emotional sense of a text and thereby analyze the emotional symbolization that underlies the local culture of organizations and communities.

The products of the ETA method have pragmatic value. The majority of our emotional tensions, particularly the most dramatic, concern the experience of being confronted with the experience of impotence. If we have the power to intervene, or even to reformulate the problem in a new way, we feel alive. Insights generated by the ETA approach can help improve organizational competence and aid people in community settings in thinking about the emotional dynamics that underlie their relationships with each other. Thus, ETA empowers both researchers and community members and their organizations in sharing and reorganizing knowledge concerning specific problems that derive from collusive dynamics, thereby promoting the growth of community competence.

\section{REFERENCES}

Benzècri, J. P. (1981). Analyse des donnée en linquistique [Analysis of data in linguistics]. Paris, France: Dunod.

Bucci, F. (2014). Cultural representations of mental illness in contemporary Japan. Rivista di Psicologia Clinica, 1, 82-108.

Carli, R., \& Pagano, P. (2008). San Lorenzo: La cultura del quartiere e $i$ rapporti con psicologia [San Lorenzo: The culture of the neighborhood and the relationships with psychology]. Rome, Italy: Kappa.

Carli, R., \& Paniccia, R. M. (1981). Psicosociologia delle organizzazioni e delle istituzioni [Psychosociology of organizations and of institutions]. Bologna, Italy: il Mulino.

Carli, R., \& Paniccia, R. M. (2002). L'analisi emozionale del testo: Uno strumento psicologico per leggere testi e discorsi [Emotional textual analysis: A psychological tool for reading texts and discourses]. Milan, Italy: Franco Angeli.

Carli, R., \& Paniccia R. M. (2003). Analisi della domanda: Teoria e tecnica dell'intervento in psicologia clinica [Analysis of the demand: Theory and technique of intervention in clinical psychology]. Bologna, Italy: il Mulino.

Fanelli, F., Terri, F., Bagnato, S., Pagano, P., Potì, S., Attanasio, S., \& Carli, R. (2006). Il rapporto di lavoro atipico: Modelli culturali, criticità e linee di sviluppo [The relationship of atypical work: Cultural models, problems and development lines]. Rivista di Psicologia Clinica, 1, 61-79.

Fornari, F. (1976). Simbolo e codice: Dal processo psicoanalitico all'analisi istituzionale [Symbol and code: From the psychoanalytic process to institutional analysis.]. Milan, Italy: Feltrinelli.

Lancia, F. (2004). Strumenti per l'analisi dei testi. Introduzione all'uso di T-LAB [Tools for text analysis. Introduction to T-LAB]. Milan, Italy: Franco Angeli.

Matte Blanco, I. (1975). The unconscious as infinite sets: An essay in bi-logic. London, England: Gerald Duckworth.

Paniccia, R. M., Di Ninni, A., \& Cavalieri P. (2006). Un intervento in un centro di salute mentale [An intervention in a mental health center]. Rivista di Psicologia Clinica, 1, 80-95.

Paniccia, R. M., Dolcetti, F., Giovagnoli, F., \& Sesto, C. (2014). La rappresentazione dell'accoglienza presso un centro di salute mentale Romano a confronto con la rappresentazione dei servizi di salute mentale in 
un gruppo di cittadini Romani: Una ricerca intervento [The representation of the reception service in a mental health center of Rome confronted with the representation of mental health services in a group of Roman citizens: An intervention-research]. Rivista di Psicologia Clinica, 1, 186-208.

Paniccia, R. M., Giovagnoli, F., Bucci, F., \& Caputo, A. (2014). Families with a child with a disability: The expectations toward services and psychology. Rivista di Psicologia Clinica, 2, 84-107.
Paniccia, R. M., Giovagnoli, F., \& Caputo, A. (2014). In-home elder care. The case of Italy: The badante. Rivista di Psicologia Clinica, 2, 60-83.

Reinert, M. (1993). Les "mondes lexicaux" et leur "logique" à travers l'analyse statistique d'un corpus de récits de cauchemars ["Lexical worlds" and their "logic" through the statistical study of a body of nightmare narratives]. Langage et Société, 66, 5-39. 
